\title{
Energy-efficient Modulation and Physical Layer Design for Low Terahertz Band Communication Channel in 5G Femtocell Internet of Things
}

\author{
Nabil Khalid ${ }^{\mathrm{a}}$, Turker Yilmaz ${ }^{\mathrm{a}, *}$, Ozgur B. Akan ${ }^{\mathrm{a}, \mathrm{b}}$ \\ ${ }^{a}$ Next-generation and Wireless Communications Laboratory (NWCL), Department of Electrical and Electronics Engineering, Koç \\ University, Istanbul, 34450, Turkey \\ ${ }^{b}$ Internet of Everything Group, Electrical Engineering Division, Department of Engineering, University of Cambridge, Cambridge, CB3 \\ OFA, United Kingdom
}

\begin{abstract}
High throughput capability of the terahertz band $(0.3-10 \mathrm{THz})$ wireless communications is expected to be utilized by the fifth generation of mobile telecommunication systems and enable a plethora of new applications. Supporting devices will transfer large amounts of data in both directions, causing high energy consumption by the electronic circuitries of the equipment in use. Therefore, physical layer for these systems must be designed carefully in order to reduce energy consumption per bit. In this paper, the best performing modulation scheme and hardware parameters that minimize the energy consumption without affecting the system throughput are determined. THz band device technologies are outlined and a complete survey of the state-of-the-art low-THz band circuit blocks which are suitable for mass market production is given. It is shown that for short-range communications, M-ary quadrature amplitude modulation is the most energy-efficient technique that can lead up to $90 \%$ reduction in consumed energy. Moreover, optimal transceiver parameters which can be used to further minimize the energy consumption of the $\mathrm{THz}$ band system are examined.
\end{abstract}

Keywords: Energy efficiency, Modulation, Physical layer, Submillimeter wave communication, 5G mobile communication, Internet of Things

\section{Introduction}

Over the last two decades mobile communications have radically progressed from just being a luxury service for business people. The subscriber number of mobile telephony passed both the 1 billion mark and its fixed-line counterpart in 2002 permanently: As of 2014, mobile device and connections reached to 7.4 billion globally, resulting a penetration rate over $100 \%$ for the first time, and expected to grow to 11.6 billion by 2021 [1]3].

Technical performance requirements of the fourth generation (4G) of mobile telecommunication systems, IMT-Advanced 4, were identified in 2008 by the report International Telecommunication Union (ITU) Radiocommunication Sector (ITU-R) M.2134 [5]. LTE-Advanced and WirelessMAN-Advanced were specified as the two complying standards on 18 January 2012, and this date can be considered to be the beginning of the standardization activities on the $5 \mathrm{G}$ systems.

There are some ever-present expectations from the next-generation of cellular systems, the foremost of which can claimed to be peak data rate increase. The highest data rate for the third generation systems was defined as 20

\footnotetext{
* Corresponding author.

Email addresses: nkhalid15@ku.edu.tr (Nabil Khalid), turkeryilmaz@ku.edu.tr (Turker Yilmaz), akan@ku.edu.tr (Ozgur B. Akan)
}

$\mathrm{Mb} / \mathrm{s}$ in 1997 by the report ITU-R M.1034-1 6], whereas, this value for the $4 \mathrm{G}$ downlink is $1500 \mathrm{Mb} / \mathrm{s}$. 5 . However, the latter assumes an extended operation bandwidth of $100 \mathrm{MHz}$ and $4 \times 4$ multiple-input multiple-output (MIMO) antenna array, though the current industry norm is 2-stream MIMO and 4-stream MIMO is only perceived to be possible by the time $5 \mathrm{G}$ telecommunication systems arise. Thus, in view of the advancement of the wireless standards, peak data rate designation of beyond $4 \mathrm{G}$ systems is expected to be on the order of $10 \mathrm{~Gb} / \mathrm{s}$.

Besides performance, mobile telecommunication market and applications are continuously evolving too. The increase in the number of user equipments (UEs) is led by smartphones, and this trend is expected to continue, as the share of smart devices among total connections is predicted to rise from its 2014 value of $26 \%$ to $74.7 \%$ by 2021 . Such an upsurge does not just alter the composition of principal mobile application and services, but also adds additional growth pressure on network capacity and connection speeds, since average data usage per smart devices range between 37 and 132 times that of a basic mobile phone, depending on the device and connection types 2,3 .

Increasing the carrier frequency, $f_{c}$, to the low end of the $\mathrm{THz}$ band has been proposed as one alternative method to meet the radio interface performance requirements of the $5 \mathrm{G}$ systems [7, 8. Research 
activities in this spectrum are increasing, as the channel characterization and semiconductor device behaviors are already being examined. However, unlike the $60 \mathrm{GHz}$ industrial, scientific and medical radio band, modulation schemes for the $\mathrm{THz}$ band communications are yet to be defined. In [9], a distance aware modulation scheme which uses distance and frequency dependent behavior of the $\mathrm{THz}$ band channel to adaptively utilize the bandwidth is proposed. An important factor in the selection of the modulation technique for the $\mathrm{THz}$ band is the higher amount of energy that is required for data transmission. Thus, minimizing the energy consumption factor is necessary to make the communications viable and cost-effective. Most of the literature on wireless communication energy consumption is focused on transmitting small amounts of data at sub 6 $\mathrm{GHz}$ bands. For example, in [10], energy consumption analysis of a communication system operating at 2.5 $\mathrm{GHz}$ is analyzed by including the transmission and circuit energy consumptions. It is shown that for short-range applications, M-ary quadrature amplitude modulation (MQAM) is more energy efficient than M-ary frequency-shift keying (MFSK).

In this paper, use cases that contain large amounts of data transmission in the low- $\mathrm{THz}$ band are investigated in order to determine the best performing and lowest energy consuming modulation methods. The analyses are extended towards optimal physical layer (PHY) parameters which can further minimize energy consumption, and to propose ideal parameters required for general $\mathrm{THz}$ band transceiver (TRX) design. The used models are also based on semiconductor technology, since silicon ( $\mathrm{Si}$ ) complementary metal-oxide-semiconductor (CMOS) is still the best choice for bulk fabrication of inexpensive products.

The remainder of this paper is organized as follows. Section 2 provides state-of-the-art $\mathrm{THz}$ device technologies that operate in room temperature and hold promise for use in commercial $5 \mathrm{G}$ products. In Section 3 , the $\mathrm{THz}$ band TRX model used for energy consumption analyses is described. Energy consumption characteristics of MQAM and MFSK are researched in Section 4. Optimization of modulation parameters, together with the comparison of the MQAM and MFSK simulation results, are provided in Section 5. Optimization of PHY parameters is examined in Section 6, and the article is concluded following a précis on associated open research issues in Section 7 .

\section{THz Band Device Technologies}

While the $\mathrm{THz}$ band seems to offer an abundant spectrum for every radio service conceivable, it actually presents a very harsh environment for electromagnetic (EM) wave propagation. Although prohibitively high attenuation by atmospheric gases is advantageous for some small number of specific applications, like intersatellite communications links because it assists those to be isolated from any possible interference from the Earth, $\mathrm{THz}$ band is yet to be utilized for communication purposes. However, this has not been the case for the whole scientific field as the $\mathrm{THz}$ band contains some unique and valuable information that are within the research interests of different areas. For example, temperatures of the interstellar dust clouds range between 10 and $200^{\circ} \mathrm{K}$, which corresponds to about 0.21 and $4.3 \mathrm{THz}$, respectively. Therefore, energy radiated from interstellar gas, which is used for star formation research, lies entirely within the $\mathrm{THz}$ band. Gases that make up the Earth's atmosphere also have thermal emission lines in the $\mathrm{THz}$ band, creating Earth science's need for measurement instruments working at $\mathrm{THz}$ frequencies.

Several device technologies are available today which jointly cover the transmitter (TX) and receiver (RX) needs of the entire $\mathrm{THz}$ spectrum, ranging from metamorphic high electron mobility transistors to quantum cascade lasers; however, only a very small percentage of these possess the potential to be used for $5 \mathrm{G}$ communication systems. If $5 \mathrm{G}$ systems are to acquire high market penetration rates, respective $\mathrm{UE}$ and network devices must be robust, lightweight, highly integrated and most importantly, low-cost. Taking into account the technologies which are currently used to manufacture the hardware of virtually all mainstream communication devices and after a review of the currently available $\mathrm{THz}$ device technologies, Si CMOS appear as the only viable candidates for $5 \mathrm{G}$ despite their shortcomings in practically all electronic performance criteria.

Compared to the III-V semiconductor compounds, Si has worse material properties than many. Lower electron mobility, smaller energy band gap and higher resistivity of Si result in devices with inferior figures of merit. CMOS, likewise, has poorer transistor and passive performances than the corresponding components produced using III-V compound processes. However, there are plenty of critical reasons that have caused $\mathrm{Si}$ and CMOS to dominate the global semiconductor market, and with the current development rate in corresponding areas, their places look secure. Si, first of all, is vastly available all around the world and its purification is simple. Mechanical characteristics of Si make it a sturdy material, thus easy to manufacture and handle. Si also has high thermal conductivity enabling efficient thermal management of devices. It is simple to form insulators with exceptional dielectric properties like silicon dioxide that are used as CMOS transistor gates among many other functions. The doping concentration of $\mathrm{Si}$ has a very high range and with the already established manufacturing capacity and continued demand, low-cost production is ensured. On the other hand, since CMOS technology is essentially the same for all devices regardless of the frequency of operation, CMOS's intrinsic advantages like integration of higher frequency circuits with baseband circuitry, digital calibration for better performance, high yield and built-in self test also holds true for the $\mathrm{THz}$ range devices. 
Initial IEEE mm-wave standards 802.11ad and 802.15.3c were ratified in December 2012 and October 2009, respectively. Furthermore, considering the fact that the first submm-wave standard, IEEE 802.15.3d, was approved in September 2017, it will be safe to say we are at least a decade away from commercial low-THz band products [11 13. Nevertheless, research activities on both circuitry and communication domains are starting to accelerate, which includes MIMO systems as well [14. Emerging $\mathrm{THz}$ band applications that also develop Si CMOS technology include imaging [15], sensors [16] and chip interconnection [17. Artificial dielectric layers are proposed to improve performance of on-chip antennas radiating at the low- $\mathrm{THz}$ band [18. However, in line with the subject of the paper, in the following subsections a complete survey is presented on the state-of-the-art Si CMOS THz circuit blocks and modules designed for communication purposes. Moreover, devices whose operation frequencies are contained by the first 3 transmission windows of the low- $\mathrm{THz}$ band, which, approximately, range from 275 to $420 \mathrm{GHz}$ [19, 20], are selected in order to demonstrate the potential for $5 \mathrm{G}$ in the $\mathrm{THz}$ region. This limitation imposed the exclusion of a number of notable Si CMOS studies that are just outside the frequency range [21, 22].

\subsection{Signal Sources}

$\mathrm{THz}$ signal source fabrication using CMOS is probably the most difficult field of the $\mathrm{THz}$ electronics research and only recently implementations with acceptable power, high stability and frequency tuning have started appearing in the scientific literature. Depending on the power gain cutoff frequency $\left(f_{\max }\right)$ of the transistors that are used, low- $\mathrm{THz}$ band can be reached through either frequency multiplication or direct generation. If the $f_{\max }$ of a device is large enough for the intended $\mathrm{THz}$ application, direct generation is commonly preferred since the power efficiency is better and smaller chip area is needed compared to frequency multiplication. However, especially for $\mathrm{Si}$ CMOS devices, this is predominantly not the case. Therefore, signal sources are specifically designed to efficiently generate power at the harmonic frequencies of built-in non-linear diodes, so that appropriate harmonics of the fundamental frequency $\left(f_{0}\right)$ can be output.

One Si CMOS source employing a triple-push architecture is reported in [23. An N-push oscillator consists of $\mathrm{N}$ coupled oscillators which use a shared resonator and output $2 \pi / N$ phase-shifted signals. When these signals are combined, $\mathrm{N}^{\text {th }}$ harmonic components are constructively added, whereas the rest, in theory, are negated. Even though this method is useful for higher frequency generation, discontinuous tuning is observed in the event of uneven phase-shift [24].

In [23, the same 2 triple-push oscillator cores are locked by magnetic coupling, and the power is conveyed to the differential ring antenna through a matching stage. The device is realized in a 65-nm CMOS process over a
$500 \times 570 \mu \mathrm{m}^{2}$ die area, with oscillators occupying $120 \times$ $150 \mu \mathrm{m}^{2}$ of the total. Output frequency is measured to be tunable from 284 to $288 \mathrm{GHz}$ by reducing the supply voltage from 1.4 to 0.7 volts $(\mathrm{V})$, and the source can generate a peak output power of $-1.5 \mathrm{dBm}$ at the upper limit of the tuning range by consuming 275 milliwatts $(\mathrm{mW})$ of DC power. The circuitry was also packaged with a Si lens on an FR-4 board, but since the aim was demonstration and did not involve original design, that part is omitted.

Another novel CMOS source that is implemented in a $65-\mathrm{nm}$ low-power bulk CMOS process is presented in 25]. For frequency tuning, placing varactors inside the $\mathrm{LC}$ resonator is a common practice that is shown to work satisfactorily up to $0.1 \mathrm{THz}$ 26]. However, at higher frequencies varactor performance degrades. The significance of the design of [25] originates from eliminating varactors from the voltage-controlled oscillator (VCO), but still delivering a frequency tunable source with high output power which functions at the beginning of the submm-wave band. By adding phase shifters to the proposed 4 core coupled oscillator system, locking frequency of the $\mathrm{VCO}$ is made adjustable in accordance with the phase shifts between each core and the respective injected signal. Even though the provided simulation result illustrated that the third harmonic generates higher current around $300 \mathrm{GHz}$, fourth harmonic is chosen also for the symmetry it brings. One of the two VCOs that are fabricated for the study radiates peak output power of $-1.19 \mathrm{dBm}$ around its $13 \mathrm{GHz}$ of tuning range centered at $290 \mathrm{GHz}$, therefore achieving the highest output power and tunability for all the oscillators available in the literature which operate in and beyond the low- $\mathrm{THz}$ band, even including the ones using compound semiconductor technologies. DC-to-RF conversion efficiency stands at $0.23 \%$ due to the $325 \mathrm{~mW}$ DC power input, and the chip is printed on an area of $600 \times 600 \mu^{2}$.

\subsection{Transmitters}

Not just sources but also complete TXs are being developed for $\mathrm{THz}$ frequencies. One latest example is a phased array [27] that is expanded over the delay-coupled oscillator method by the authors of [25]. The idea of controlling the oscillator frequency through the phase shift between adjacent cores works on a one-dimensional ring. To extend this effect over two dimensions, a $2 \times 2$ central loop is connected to 4 other similar loops solely through one of its vertices, creating a $4 \times 4$ coupled array. Adjacent nodes are situated at a fixed distance which equals half of the radiation wavelength, and the oscillators are linked with phase shifters. Patch antennas are used for radiation to prevent substrate coupling. A sample, likewise [25], is manufactured in a $65-\mathrm{nm}$ bulk CMOS process over an area of $1.95 \times 2 \mathrm{~mm}^{2}$. Peak total radiated power is measured at $338 \mathrm{GHz}$ as $0.8 \mathrm{~mW}$, or $-0.97 \mathrm{dBm}$, and equivalent isotropically radiated power (EIRP) as $51 \mathrm{~mW}$, or $17.1 \mathrm{dBm}$, using $1.54 \mathrm{~W}$ of $\mathrm{DC}$ power. $12 \mathrm{~dB}$ of the 
$18 \mathrm{~dB}$ antenna directivity is due to array gain, and the rest due to patch antenna directivity. Beam steering is feasible across $45^{\circ}$ in azimuth $(\Phi)$, and $50^{\circ}$ in elevation $(\Theta)$ angles. Center frequency tuning measurements are performed between 337 and $339 \mathrm{GHz}$. However, the authors claim that $2.1 \%$ tuning is possible via altering the coupler supply voltage, which results in a $7.1 \mathrm{GHz}$ spectrum around $338 \mathrm{GHz}$.

Another architecture is tunable at second harmonic frequencies which are between 276 and $285 \mathrm{GHz}$ [28. Distributed active radiator (DAR) 29] and inverse design approach lies at the core of this design. Typically, power generation and radiation are implemented by different circuit blocks. However, in 28, surface currents on Si chip metal layers are first calculated for a specific EM field, and then synthesized using a DAR, which is made of 4 cross-coupled transistor pairs located symmetrically along 2 loops that are shaped into a Möbius strip. This way, second harmonic signal is radiated, while fundamental and other harmonic signals are filtered. The implementation consists of 16 DAR cores in a $4 \times 4$ array, and it is realized in a $45-\mathrm{nm}$ CMOS silicon on insulator process. The output of the center VCO, which is tunable from 91.8 to $96.5 \mathrm{GHz}$ using a $1.1 \mathrm{~V}$ supply voltage, is distributed to 4 separate divide-by-two frequency dividers that generate quadrature in-phase (I) and quadrature (Q) signals. Signal is then transferred through phase rotator and frequency triplers to drive the DARs. The resulting circuit, which has a chip area of $2.7 \times 2.7 \mathrm{~mm}^{2}$, is capable of beam steering nearly $80^{\circ}$ in both $\Theta$ and $\Phi$ planes and provides an EIRP of 9.4 $\mathrm{dBm}$ at $280.8 \mathrm{GHz}$ via the $16 \mathrm{dBi}$ maximum directivity.

\subsection{Transceivers}

The final integrated TRX model [30] is included to provide a TRX example, even though the device is implemented in 130-nm silicon-germanium bipolar CMOS process. Moreover, its 367 to $382 \mathrm{GHz}$ working range is around an atmospheric attenuation local maximum, thus making the device unsuitable for communication purposes. The TRX design is a homodyne frequency-modulated continuous wave radar using triangular modulation signal. Differential Colpitts VCO generates the fundamental signal at $92.7 \mathrm{GHz}$ with $8.3 \%$ tuning radius, which is followed by drive amplifiers. Inside the TX, initially, balanced quadrature I and Q signals are coupled through transformers to the frequency quadrupler. 2 push-push pairs compose the quadrupler, which outputs the fourth harmonic frequency, and separate patch antennas, each containing 2 patches, radiates and receives the signal. On the RX side a subharmonic mixer, driven by second harmonic quadrature I and Q signals, down-converts to intermediate frequency (IF), before the concluding IF amplifier stage. The TX translates $3 \mathrm{dBm}$ VCO output power into -14 to $-11 \mathrm{dBm}$ EIRP, the RX noise figure $\left(N_{f}\right)$ is assessed to be between 35 and $38 \mathrm{~dB}$, and the entire TRX consumes $380 \mathrm{~mW}$ power above a total space of $2.2 \times 1.9$ $\mathrm{mm}^{2}$. Also, a full subharmonic Schottky diode based TRX

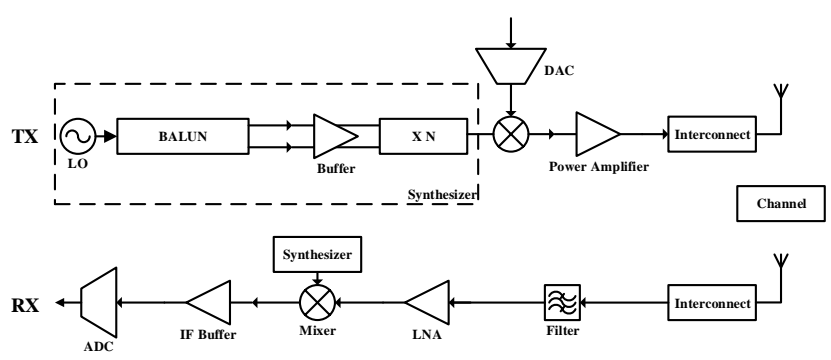

Figure 1: Architecture of the $\mathrm{THz}$ band transceiver.

architecture that provides a bandwidth of $20 \mathrm{GHz}$ which is extendible up to $40 \mathrm{GHz}$ is shown in 31.

\section{3. $\mathrm{THz}$ Band Communication System Model}

We modeled a communication link between $2 \mathrm{THz}$ band nodes in order to analyze the energy consumption of a $\mathrm{THz}$ band wireless system. A generic wireless communications system can be decomposed into 5 main components: TX, RX, synthesizer, power amplifier (PA) and antenna. All signal processing blocks need to be considered in the energy consumption analysis too. $\mathrm{THz}$ band signal generation requires a special synthesizer, and thus it should also be examined in detail. Contrariwise, bandwidth in the $\mathrm{THz}$ spectrum is abundant and the small electrical length enables highly directive communication. Therefore, complicated signal processing techniques such as multiuser detection or time division multiplexing are not very advantageous and hence neglected in our model. The resulting system model of the $\mathrm{THz}$ band TRX is shown in Fig. 1] This system can easily be modified to analyze the energy consumption in a full-dimension MIMO (FD-MIMO) based on the system shown in 32 .

$\mathrm{THz}$ band signal synthesizer mainly consists of a local oscillator (LO), balun, buffer amplifier (BA) and multiplier 33. The multiplying factor depends on the generated frequency. The baseband digital signal is first converted to analog signal using a digital-to-analog (DAC) converter. This signal modulates the frequency synthesizer signal in the mixer. Subsequently, the modulated signal is amplified using a PA and transmitted into the channel through the antenna. Usually, antennas in the $\mathrm{THz}$ band devices are connected to the circuit using special interconnects whose power dissipation is considerable and cannot be neglected. On the RX side, the $\mathrm{THz}$ signal is first filtered inherently by the interconnect and then amplified by the low noise amplifier (LNA), downconverted by the mixer and amplified by the IF buffer. Finally, the IF signal is converted back to digital signal using an analog-to-digital converter (ADC).

TRX is assumed to be operating on a hybrid mode basis. When there is a signal to transmit, all the TRX components operate in active mode and consume full power. Conversely, when there is no signal to transmit, 
TRX switches to inactive mode, which saves energy. When switching from the inactive mode to the active mode there is a transient mode, which is the time required to turn all the components into the active state.

To calculate the power consumption, a simplified case of wireless communication is considered where one of the nodes has $I$ bits to transfer in time $T$. TX turns on for active mode time, $T_{a c t}$, to transmit these bits and then returns back to the inactive mode. $T_{\text {act }}$ parameter needs to be optimized based on the modulation scheme. The transmission period consists of $T=T_{a c t}+T_{\text {settl }}+$ $T_{\text {off }}$, where, $T_{\text {settl }}$ is the transient time whose duration depends mainly on the phase lock loop settling time in the frequency synthesizer and $T_{\text {off }}$ is the inactive mode duration. In this scenario, the total energy consumption, $E_{\text {total }}$, required to send $I$ bits can be written as

$$
E_{\text {total }}=P_{a c t} T_{a c t}+P_{o f f} T_{o f f}+P_{\text {settl }} T_{\text {settl }}
$$

where the active mode power, $P_{a c t}$, is composed of the transmitted power and the circuit power consumption. Power consumed by LO, balun, BA, mixer, LNA, filter, IF buffer, ADC, DAC and PA are all included in the circuit power use. The power consumption of PA is $P_{a m p}=\alpha P_{t}$, where $\alpha=(\xi / \eta)-1, \xi$ is the peak-to-average power ratio (PAPR) which depends on the modulation scheme, and $\eta$ is the drain efficiency. Thus, the corresponding active mode power can be expressed as

$$
P_{a c t}=P_{t}+P_{a m p}+P_{c t}+P_{c r}
$$

where $P_{c t}$ and $P_{c r}$ are the circuit power consumptions at the TX and RX, respectively. Moreover, $P_{c t}=P_{L O}+$ $P_{\text {balun }}+P_{b u f f e r}+P_{\times N}+P_{m i x}+P_{D A C}+P_{\text {interconnect }}$ and $P_{c r}=P_{L O}+P_{b a l u n}+P_{b u f f e r}+P_{\times N}+P_{m i x}+P_{L N A}+$ $P_{\text {interconnect }}+P_{I F B u f f e r}+P_{A D C}+P_{f i l}$.

The inactive mode power consumption is orders of magnitude smaller than the other 2 states and can be neglected in the current comparison 34. In contrast, when turning on the TX, first the LO is activated until it settles and then the other circuitries are started. Hence, the transient state power depends on the power consumed by the LO at the TX and RX, which can be approximated to $P_{\text {settl }} \approx 2 P_{L O}$.

Using these defined parameters, energy consumption per information bit, $E_{b}=E_{\text {total }} / I$, is computed as

$$
E_{b} \approx \frac{(1+\alpha) E_{t}+P_{c} T_{a c t}+2 P_{L O} T_{\text {settl }}}{I}
$$

where $E_{t}=P_{t} T_{a c t}$ is the energy transmitted, and $P_{c}=$ $P_{c t}+P_{c r}$ is the circuit power consumption, whereas $P_{L O}$ and $T_{\text {settl }}$ are constants [35]. Here, the optimal $T_{a c t}$ needs to be determined to obtain the ideal constellation size for any modulation scheme. From (3), $E_{b}$ is directly proportional to the PAPR of the modulation technique. Thus, the modulation scheme must be selected carefully. In addition, it has been shown that the energy per bit increases as the bandwidth efficiency rises, which is defined by $B_{e}=I / B T_{a c t}$, where $B$ is modulation bandwidth 35 .

Employing a log-distance path loss model, the transmitted power, $P_{t}$, can be defined as

$$
P_{t}=P_{r} d^{\gamma} M_{l} G_{1}
$$

where $P_{r}$ is the received power, $d$ is the link distance, $\gamma$ is the path loss exponent, $M_{l}$ is the link margin compensation used to account for any variations in the system and $G_{1}$ is the gain factor for unit distance, which includes the gains of the TX and RX antennas and the path loss at $1 \mathrm{~m}$. For an indoor office environment, $\gamma$ can be assumed 4 36. Furthermore, the free-space path loss for $1 \mathrm{~m}$ at $300 \mathrm{GHz}$ is approximately $82 \mathrm{~dB}$ and since an antenna gain of $22 \mathrm{dBi}$ is already demonstrated [37], we utilized $G_{1}$ as $38 \mathrm{~dB}$ in our analyses.

\section{Energy Consumption Analyses of MQAM and MFSK Modulations}

Bandwidth is not scarce in the $\mathrm{THz}$ spectrum. Therefore, to transmit large amounts of data, the most energy efficient modulation scheme should be utilized. To find the optimum modulation method, the model given in the Section 3 is evaluated for the MQAM and MFSK 38. Both techniques are analyzed over an additive white Gaussian noise (AWGN) channel.

\section{1. $M Q A M$}

For a communication system that uses MQAM, the number of symbols required to send $I$ bits are $I_{s}=I / b$, where $b=\log _{2} M$ is the number of bits assigned per symbol. Moreover, $I_{s}$ can also be represented in terms of the symbol period $T_{s}$, such as $I_{s}=T_{a c t} / T_{s}$. If the system utilizes square pulses, then $T_{s} \approx 1 / B$ and hence the relationship can be expressed as

$$
b \approx \frac{I}{B T_{a c t}}=B_{e}
$$

The required $P_{r}$ for a specific set of constellation size, $N_{f}$, bandwidth and probability of error for MQAM operating in an AWGN channel is also determined using the upper bound on the error probability given by

$$
P_{r}=\frac{4}{3} N_{f} B \sigma^{2}\left(2^{b}-1\right) \ln \left[\frac{4\left(1-\frac{1}{\sqrt{2^{b}}}\right)}{b P_{b}}\right]
$$

where $\sigma^{2}$ is the power spectral density of the AWGN channel and $P_{b}$ is the bit error probability [36].

For MQAM, PAPR is calculated using $\xi=3(\sqrt{M}-$ 1) $/(\sqrt{M}+1)$ and $M=2^{I / B T_{a c t}}$. Finally, by substituting all the necessary parameters for MQAM in (3), energy per 
Table 1: System parameters

\begin{tabular}{lclc}
\hline Parameter & Value & Parameter & Value \\
\hline$B$ & $1 \mathrm{GHz}$ & $\gamma$ & 4 \\
$\eta_{M Q A M}$ & 0.15 & $\eta_{M F S K}$ & 0.35 \\
$G_{1}$ & $38 \mathrm{~dB}$ & $M_{l}$ & $40 \mathrm{~dB}$ \\
$N_{f}$ & $10 \mathrm{~dB}$ & $f_{c}$ & $300 \mathrm{GHz}$ \\
$\sigma^{2}$ & $-174 \mathrm{dBm} / \mathrm{Hz}$ & $I$ & $200 \mathrm{Mb}$ \\
$T_{\text {settl }}$ & $10 \mu \mathrm{s}$ & $T_{\text {MQAM }}$ & $100 \mathrm{~ms}$ \\
$T_{M F S K}$ & $1.07 \mathrm{~s}$ & $P_{b}$ & $10^{-3}$ \\
$P_{L N A}$ & $6.75 \mathrm{~mW}$ & $P_{\text {maxt }}$ & $53 \mathrm{~mW}$ \\
$P_{L O}$ & $100 \mathrm{~mW}$ & $P_{\text {fil }}$ & $1 \mathrm{~mW}$ \\
$P_{A D C}$ & $1.4 \mathrm{~W}$ & $P_{\text {mix }}$ & $7.9 \mathrm{~mW}$ \\
$P_{D A C}$ & $2.5 \mathrm{~mW}$ & $P_{\text {balun }}$ & $1.2 \mathrm{~mW}$ \\
$P_{I F B u f f e r}$ & $5 \mathrm{~mW}$ & $P_{\text {buffer }}$ & $8 \mathrm{~mW}$ \\
$P_{\times N}$ & $13 \mathrm{~mW}$ & $P_{\text {interconnect }}$ & $1.8 \mathrm{~mW}$ \\
\hline
\end{tabular}

bit is expressed as

$$
\begin{gathered}
E_{b}=\frac{4}{3}(1+\alpha) N_{f} \sigma^{2}\left(2^{b}-1\right) \frac{G_{1}}{b} \ln \left[\frac{4\left(1-\frac{1}{\sqrt{2^{b}}}\right)}{b P_{b}}\right] \\
+\frac{P_{c} T_{a c t}+2 P_{L O} T_{\text {set } t}}{I}
\end{gathered}
$$

System parameters for the $\mathrm{THz}$ band TRX are given in Table 139 41. The DAC and ADC are also assumed to be the Texas Instruments Inc. models DAC1220 and ADC08D500, respectively. Due to high PAPR, MQAM requires linear $\mathrm{PA}$. Amplification in the $\mathrm{THz}$ band is hard to achieve using solid state devices due to their low transistor cut off frequency. Hence, class-A PA is selected whose efficiency is $\eta_{M Q A M}=0.15$ [4]. $I$ is found using $b_{\min }=L / B T$ and the fact that minimum constellation size, $b_{\text {min }}$, equals 2 . Here, $B$ and $T$ are chosen for the specific case of Internet of Things.

To determine the optimum active time for transmission at different $d, E_{b}$ is plotted against the normalized $T_{a c t}$ in Fig. 2. For low $d$, the energy consumption is less for smaller $T_{\text {act }}$. Hence, significant amount of energy can be saved by optimum $T_{\text {act }}$.

\section{2. $M F S K$}

In MFSK, the number of orthogonal carriers equals the constellation size $M$, with a minimum intercarrier separation of $1 / 2 T_{s}$. Hence, the total bandwidth is $B \approx$ $2^{b} / 2 T_{s}$, and the bandwidth efficiency is $B_{e}=2 b / 2^{b}$. By comparing the bandwidth efficiency of MFSK with the general bandwidth efficiency equation $B_{e}=I / B T_{a c t}$, the following equation for $T_{a c t}$ in MFSK is obtained

$$
T_{a c t}=\frac{2^{b} I}{B 2 b}
$$

The relation between required $P_{r}$ for a given probability of error, bandwidth, constellation size and $N_{f}$

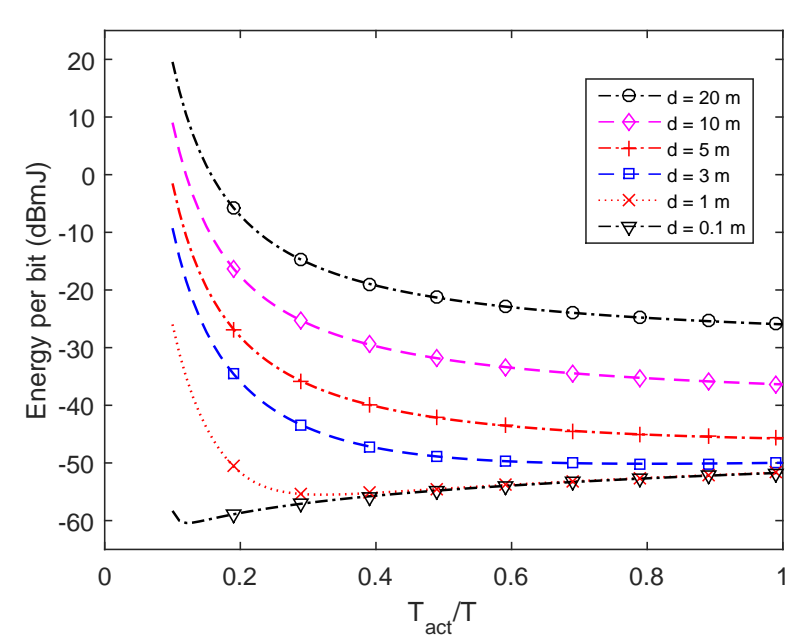

Figure 2: Energy consumption per information bit at various distances vs. normalized $T_{a c t}$ for the MQAM system.

for MFSK can be determined using the upper bound on error probability, which is calculated as 36]

$$
P_{r}=4 N_{f} \sigma^{2} \frac{2 B}{2^{b}} \ln \left(\frac{2^{b-2}}{P_{b}}\right)
$$

Unique nature of MFSK modulation requires some modification in the circuit parameters and model. In MFSK, $\xi=1$ due to the constant signal amplitude. This allows the amplifier to be used in saturation and achieve maximum efficiency. Furthermore, it allows the use of non-linear amplifiers, such as class-B amplifiers that provide better drain efficiency 42 . Hence $\eta=0.35$ is kept for the $\mathrm{THz}$ band PA. Conversely, in the TX, the mixer and DAC need to be removed since frequency modulation is carried out directly in the synthesizer. When all the required MFSK parameters are placed in (3), $E_{b}$ becomes

$$
E_{b}=4(1+\alpha) N_{f} \sigma^{2} \frac{G_{d}}{b} \ln \left(\frac{2^{b-2}}{P_{b}}\right)+\frac{P_{c} T_{a c t}+2 P_{L O} T_{\text {settl }}}{I}
$$

where $G_{d}$ is the power gain factor, and the bandwidth and packet size for MFSK are kept similar to the MQAM configuration. However, the maximum time delay, $T_{M F S K}$, was changed to $1.07 \mathrm{~s}$, which restricts the maximum value of $b$ to 6 . Unlike MQAM, MFSK modulation is less bandwidth efficient and thus it requires more transmission time for the same number of bits and a given bandwidth.

$E_{b}$ for MFSK modulation scheme is shown in Fig. 3. For low $d$, smaller $b$ value is more energy efficient, and energy consumption rises with growing $b$. This is due to the effect of circuit power consumption, which increases for higher $b$. Yet, for distances higher than $10 \mathrm{~m}$, transmission power dominates the circuit power consumption and thus larger $b$ becomes more energy efficient. In an AWGN channel, the larger the $M$ is, the more energy efficient MFSK becomes, leading to our result of reduction in 


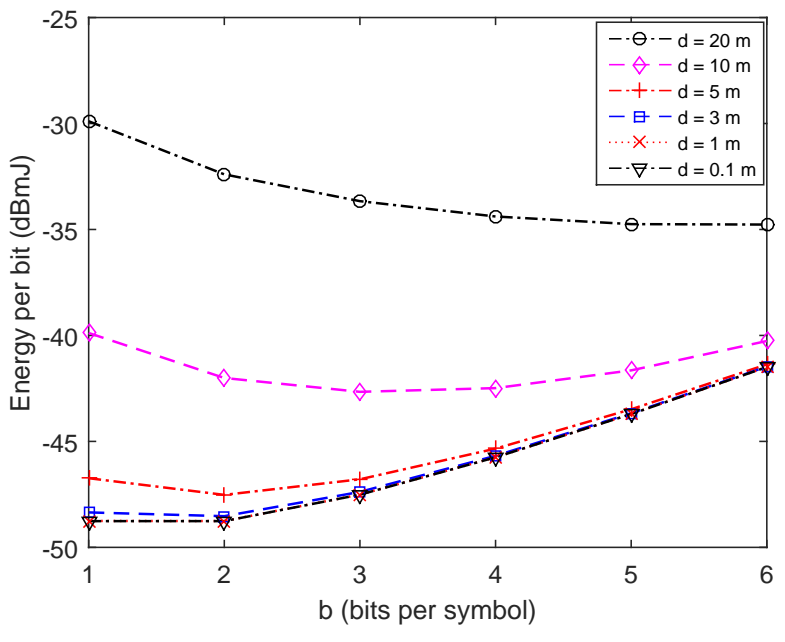

Figure 3: Energy consumption per information bit at various distances vs. bits per symbol (b) for the MFSK system.

transmission energy at higher $d$. To illustrate, about $50 \%$ energy saving is achieved for $b=4$ in the $d=10 \mathrm{~m}$ case.

\section{Modulation Parameters Optimization}

MQAM is a bandwidth efficient modulation scheme, requiring less time to transmit a given amount of data. The energy consumption analysis of MQAM in the $\mathrm{THz}$ band shows that for $d<1 \mathrm{~m}, T_{a c t}$ should be small enough to reduce the energy consumption. However, for $d>3 \mathrm{~m}$, a higher $T_{a c t}$ will decrease $E_{b}$. This is due to the fact that at longer distances, transmission power outweighs the effect of circuit power. Hence, for $d>3 \mathrm{~m}$, optimizing $T_{a c t}$ does not help with energy saving, and therefore the maximum value of $T_{\text {act }}$ should be used.

MFSK is less efficient in terms of transmission time, and therefore the time required to send a certain amount of bits is higher. But, the constant envelope $(\xi=1)$ significantly relaxes the PA linearity requirement and thus a power efficient transmission can be achieved. The energy consumption analysis of MFSK in the $\mathrm{THz}$ band shows that for $d<7 \mathrm{~m}, b=2$ is the optimum choice. Whereas, for $d>7 \mathrm{~m}$, higher $b$ value yields better energy efficiency.

A comparison of TRX energy consumption using MQAM and MFSK modulation schemes is shown in Fig. 4. For each $d$ in the MQAM TRX, optimum $T_{a c t}$ value is used to minimize energy consumption. These parameters are obtained from the energy per bit analysis of MQAM. For example, for the $d=0.1 \mathrm{~m}$ case, $T_{a c t}=12 \mathrm{~ms}$ is used and for $d=4 \mathrm{~m}, T_{a c t}=100 \mathrm{~ms}$ is employed. Similarly, optimum $b$ is selected during the MFSK modulation energy consumption analysis. The comparison of both modulation techniques show that for $d<4 \mathrm{~m}$, MQAM requires less $E_{b}$, given optimum $T_{a c t}$ is used. However, for $d>4 \mathrm{~m}$, MFSK is more efficient and hence consumes less energy than MQAM for the low-THz band operation. The

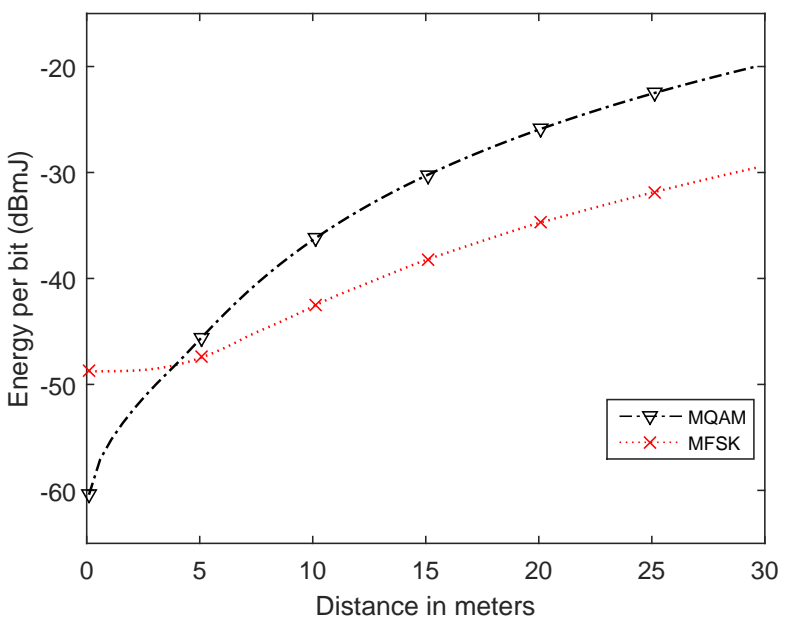

Figure 4: Energy consumption per information bit at various distances for the optimized MQAM and MFSK systems.

optimized parameters for the MQAM and MFSK systems operating at different $d$ are given in Table 2 .

The potential usage models for $\mathrm{THz}$ band can be divided into 3 main categories. The first category is very short distance communication which includes applications such as gadgets connected to a computer, ultra high definition (UHD) displays, ultra fast portable data drives and mobile data synchronization. In these applications, the distance between the TX and RX is usually less than $2 \mathrm{~m}$. Hence, to minimize the energy consumption of the information transmission, we propose the utilization of MQAM, which can lead to greater than $90 \%$ energy saving. The second category is $\mathrm{THz}$ band $5 \mathrm{G}$ indoor wireless communications, that will enable femtocells with ultra high speed communications for a coverage range of $10 \mathrm{~m}$. Specific usage models include UHD multimedia streaming, UHD video conferencing and ultra-high speed wireless data transfer. Due to the higher mobility and distance requirements in these applications, MFSK is propositioned for adoption. The third category is wireless backhaul that operates over distances greater than $10 \mathrm{~m}$. Like the second category, the characteristics of this application also necessitates the use of MFSK [43 46].

\section{PHY Parameters Optimization}

PHY consists of the main communication hardware and is the most complex layer of any communication architecture. Optimizing the parameters of PHY can significantly reduce energy consumption in a communication link. Hence, we analyzed the effects of PHY on energy consumption. The key components of PHY are solid state circuits, amplifiers, antenna gains, RX noise, bit error rate and path loss. By examining their effect on the energy consumption, optimal hardware parameters which will reduce it can be determined. To observe the behavior of all major parameters, the values 
Table 2: Optimized parameters for the MQAM and MFSK systems at various distances

\begin{tabular}{|c|c|c|c|c|c|c|c|c|c|c|}
\hline \multirow{2}{*}{ Parameter } & \multicolumn{2}{|c|}{$\mathrm{d}=0.1 \mathrm{~m}$} & \multicolumn{2}{|c|}{$\mathrm{d}=1.1 \mathrm{~m}$} & \multicolumn{2}{|c|}{$\mathrm{d}=5.1 \mathrm{~m}$} & \multicolumn{2}{|c|}{$\mathrm{d}=10.1 \mathrm{~m}$} & \multicolumn{2}{|c|}{$\mathrm{d}=25.1 \mathrm{~m}$} \\
\hline & MQAM & MFSK & MQAM & MFSK & MQAM & MFSK & MQAM & MFSK & MQAM & MFSK \\
\hline$b_{o p t}$ & 16.66 & 2 & 5.88 & 2 & 2 & 2 & 2 & 3 & 2 & 6 \\
\hline$T_{a c t}(\mathrm{~s})$ & 0.012 & 0.2 & 0.034 & 0.2 & 0.1 & 0.2 & 0.1 & 0.26 & 0.1 & 1.067 \\
\hline$E_{b}(\mathrm{~nJ})$ & 0.909 & 13.29 & 3.04 & 13.31 & 27.94 & 18 & 238.3 & 55.47 & 5609.1 & 652.47 \\
\hline Rate (Gbps) & 16.66 & 1 & 5.88 & 1 & 2 & 1 & 2 & 0.75 & 2 & 0.1875 \\
\hline
\end{tabular}

Table 3: Normalized system parameters

\begin{tabular}{lcccccc}
\hline \multirow{2}{*}{ Parameter } & \multicolumn{6}{c}{ Normalized Value } \\
\cline { 2 - 7 } & 0 & 0.2 & 0.4 & 0.6 & 0.8 & 1 \\
\hline Amplifier effic. $(\eta, \%)$ & 0 & 20 & 40 & 60 & 80 & 100 \\
Link margin $\left(M_{l}, \mathrm{~dB}\right)$ & 40 & 32 & 24 & 16 & 8 & 0 \\
Path loss exponent $(\gamma)$ & 8 & 6.6 & 5.2 & 3.8 & 2.4 & 1 \\
Noise figure $\left(N_{f}, \mathrm{~dB}\right)$ & 20 & 16 & 12 & 8 & 4 & 0 \\
\hline
\end{tabular}

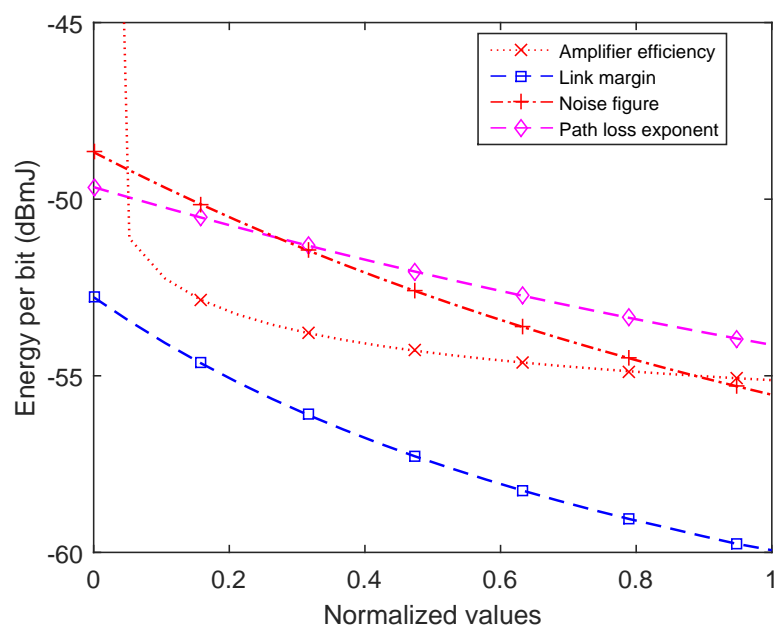

Figure 5: PHY components parameter sweep on normalized scale vs. energy consumption per bit of the MQAM system for $d=1.5 \mathrm{~m}$.

of the hardware are normalized between 0 and 1, i.e., from worst to best, respectively. The corresponding values are shown in Table 3. To provide an example, the normalized value 0 of an amplifier corresponds to $0 \%$ efficiency, whereas 1 corresponds to $100 \%$ efficiency. Furthermore, a higher efficiency means an expensive amplifier design. Therefore, any parameter with a normalized value close to 1 will perform better, but at the expense of higher cost and complexity; whereas, lower normalized value might consume higher energy, but the implementation would be easier. The comparison of MQAM parameters for $d=1.5$ $\mathrm{m}$ and MFSK parameters for $d=10 \mathrm{~m}$ are shown in Fig. 5 and Fig. 6, respectively.

Solid state circuits working in the $\mathrm{THz}$ frequency range are rapidly being developed and considered as the core technology that will enable $\mathrm{THz}$ band wireless communications. Many successful TRXs have been

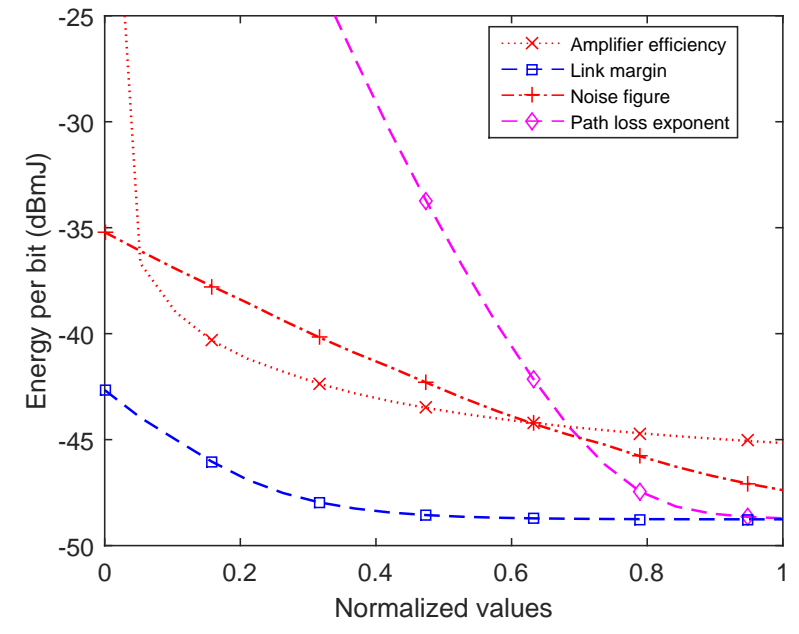

Figure 6: PHY components parameter sweep on normalized scale vs. energy consumption per bit of the MFSK system for $d=10 \mathrm{~m}$.

published in recent years [33, 47]. However, the amplifiers operating in the $\mathrm{THz}$ band require further improvement since their efficiency values are not sufficient. As power consumption of PA dominates circuit power consumption after a certain distance, their higher efficiency can improve the energy consumption for the $\mathrm{THz}$ band communications 42]. The non-linear behavior of $\eta$ can be observed from Fig. 5. In the MQAM system, $15 \%$ efficient amplifier consumes a significant amount of energy and is graded as very poor. Moreover, the non-linearly decreasing behavior depicts that after $40 \%$ efficiency, there is not considerable energy saving in MQAM. In contrast, for MFSK, PA efficiency of $60 \%$ is adequate as higher efficiency does not reduce the energy consumption substantially.

Small aperture size at high frequency allows the use of highly directional antennas that can reduce path loss. To observe the effect of antenna gain, link margin is swept from 40 to $0 \mathrm{~dB}$, which corresponds to an increase in antenna gain from 22 to $42 \mathrm{dBi}$. It is obvious that MQAM is effected by the change in power transmitted, hence in Fig. 5 the behavior of $M_{l}$ variation is linear. Furthermore, MFSK is prone to power variations, and after a certain value of $M_{l}$, any significant reduction in energy consumption is not achieved. This is confirmed by the non-linear behavior of $M_{l}$ illustrated in Fig. 6. Thus, the higher the antenna gain, the better the performance 
Table 4: Optimized PHY parameters for the MQAM and MFSK systems for 1 and $3 \mathrm{~dB}$ back-off points

\begin{tabular}{lccccc}
\hline \multirow{2}{*}{ Parameter } & \multicolumn{2}{c}{$\mathbf{1} \mathbf{~ d B}$ back-off } & & \multicolumn{2}{c}{$\mathbf{3} \mathbf{~ d B}$ back-off } \\
\cline { 2 - 3 } \cline { 5 - 6 } & MQAM & MFSK & & MQAM & MFSK \\
\hline$\eta(\%)$ & 40 & 65 & & 20 & 42 \\
$M_{l}(\mathrm{~dB})$ & 8 & 30 & & 17 & 31 \\
$\gamma$ & 3 & 2.4 & & 4 & 2.8 \\
$N_{f}(\mathrm{~dB})$ & 4.2 & 3.15 & & 7.5 & 5.2 \\
\hline
\end{tabular}

of an MQAM communication system. Whereas, for the case of MFSK, $34 \mathrm{dBi}$ would be an optimum gain value for both the TX and RX.

The path loss exponent $\gamma$ has vast effects on a communication link. It is a function of $f_{c}$, obstructions, environment and weather conditions, among others. It can range from 1.4 to 8 . Usually $\gamma$ for a furnished room is considered as 4 . To observe its effect on energy consumption, $\gamma$ is changed from 8 to 1 , which corresponds to 0 and 1 on normalized scale, respectively. In Fig. 5 , the behavior of $\gamma$ is approximately linear for the MQAM system. However, in MFSK $\gamma$ behaves non-linearly, as shown in Fig. 6, and hence higher value of $\gamma$ increases energy consumption rapidly. Moreover, the plot shows that for the MFSK system to perform well, $\gamma$ needs to be smaller than 2.4 .

$N_{f}$ is the amount of noise introduced by the RX front end to the signal received. It depicts the degradation in signal-to-noise ratio caused by the hardware components of the RX. The major components affecting $N_{f}$ are the antenna interconnects and LNA. The attenuation causing noise in the interconnects can be reduced by designing high quality interconnects that attach the antenna to the circuit, while also assisting with the out of band signal rejection. Also, the noise of amplifier can be reduced by selecting proper transistor technology and stability network of the amplifier. The ideal $N_{f}$ of a RX front end is $0 \mathrm{~dB}$ which corresponds to 1 on our normalized scale; whereas, $20 \mathrm{~dB}$ is considered to be the worst case. The behavior of $N_{f}$ is approximately linear in both Fig. 5 and Fig. 6. Hence, the lower the noise is, the less $E_{b}$ will be.

To find the optimal PHY parameter values for the least energy consumption in the $\mathrm{THz}$ band, the model is evaluated with 1 and $3 \mathrm{~dB}$ back-off values for all the parameters. $1 \mathrm{~dB}$ back-off point corresponds to the parameter value that consumes $1 \mathrm{~dB}$ less energy than the energy consumed at the ideal state, i.e., for the normalized value of 1 . This is calculated as $\left|E_{b 1}\right|-1$, where $\left|E_{b 1}\right|$ is $E_{b}$ with the parameter having its ideal value. Similarly, $3 \mathrm{~dB}$ back-off point corresponds to the parameter value that consumes $3 \mathrm{~dB}$ less energy than the energy consumed at the ideal state and is computed by $\left|E_{b 1}\right|-3$. These optimum values for the MQAM and MFSK systems are given in Table 4.

The total energy consumption per bit for optimized modulation parameters and PHY with $1 \mathrm{~dB}$ back-off point over different $d$ is shown in Fig. 7a. Optimized PHY parameters save significant amount of energy and can increase the distance where MFSK performs better than MQAM. For the stated parameters, MQAM is more efficient in terms of energy consumption till $143 \mathrm{~m}$, and after that MFSK becomes more energy efficient. Yet, in this situation the hardware parameters are very stringent and expensive. The total energy consumption per bit for $3 \mathrm{~dB}$ back-off point is shown in Fig. 7b. These PHY parameters save significant amount of energy too, and MQAM is more energy efficient for $d<12 \mathrm{~m}$.

As previously discussed, higher normalized value leads to a more expensive circuit. To compare the values of both of the modulation schemes, the average normalized parameter is calculated using

$$
\Theta=\frac{1}{N} \sum_{i=1}^{N} X_{i}
$$

where $X_{i}$ is the normalized value of the parameter $i$. Average normalized values of the communication system operating with $1 \mathrm{~dB}$ back-off point are calculated as 0.6725 and 0.6356 for MQAM and MFSK, respectively. Whereas, for $3 \mathrm{~dB}$ back-off point, these average values are 0.4925 and 0.53125 , again, for MQAM and MFSK. This shows that the $3 \mathrm{~dB}$ back-off PHY components of MQAM is on average both cheaper and more energy efficient than the MFSK. Since the $\mathrm{THz}$ band is expected to operate in femtocells where $d$ will be tens of meters, MQAM is deduced to be the superior modulation scheme for future $\mathrm{THz}$ band communications.

\section{Open Research Issues}

Number of bits being utilized per unit area is growing exponentially. Data usage is expected to increase by a thousandfold in 5G. Supporting the required amount of energy can become exigent if not handled properly. Adaptive modulation that saves energy is definitely useful, however, it is not sufficient to minimize energy consumption by itself. Other methods should also be inspected. One of the candidate techniques is reflecting antennas. It reduces the power consumption at the remote device by up to 100 times. In reflecting antennas technology, the mobile device reflects wireless signals instead of using regular TXs to generate them. This eliminates the need of PA in the UE, but consumes more energy at the base station [48].

Another interesting research topic that can significantly reduce power consumption is full-duplex wireless communications. In a full-duplex system, transmission and reception is carried out simultaneously which makes the detection of received signal very difficult. This is because the signal from the local transmitting antenna is immensely stronger than the transmission from other the node. The predicament can be handled by using 


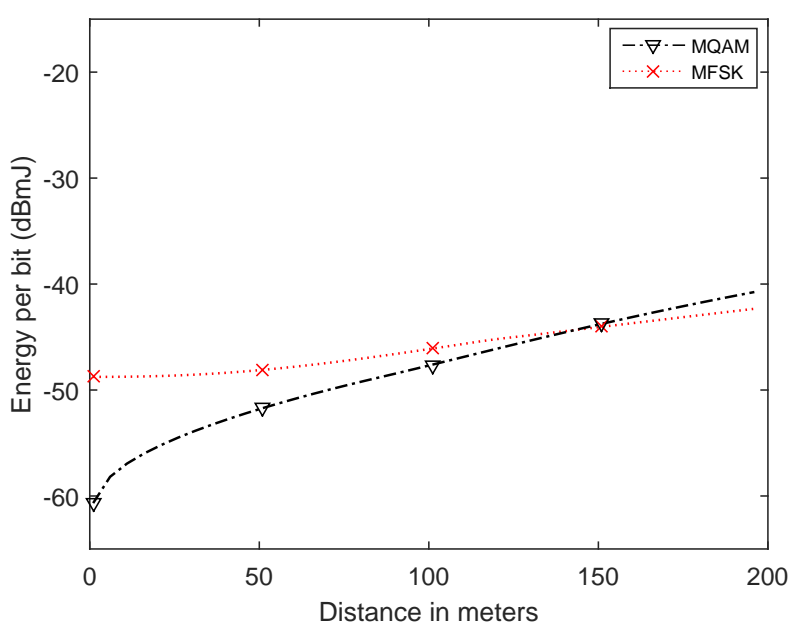

(a)

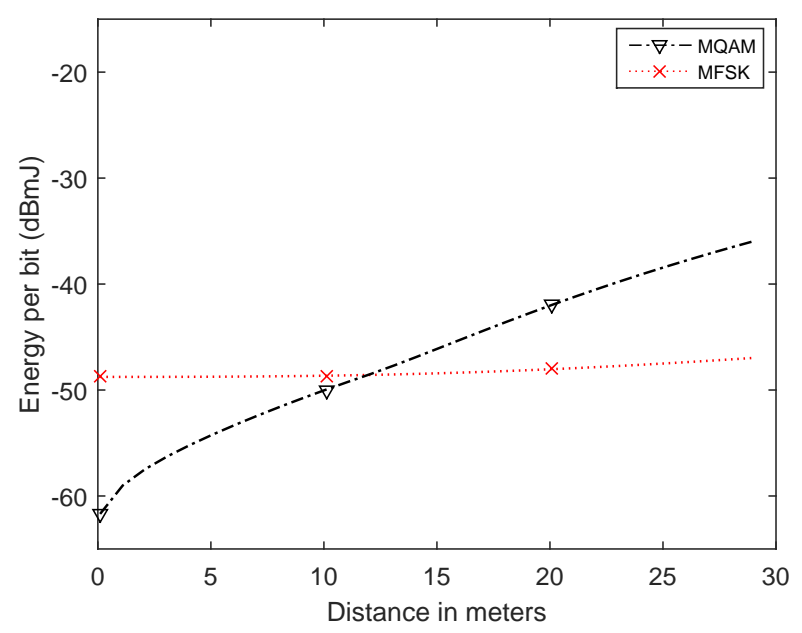

(b)

Figure 7: Energy consumption per bit for MQAM and MFSK PHY parameters optimized at (a) $1 \mathrm{~dB}$, and (b) 3 dB back-off points.

a signal inversion cancellation method [49]. Full-duplex systems reduce the time of communication, and hence the energy usage. By introducing full-duplex transmission in the $\mathrm{THz}$ band for $5 \mathrm{G}$ communication applications, $E_{b}$ can be reduced significantly with improved system latency whereas the data throughput can be doubled [50].

\section{Conclusion}

In this article, the total energy consumption of a wireless communication system operating in the $\mathrm{THz}$ band channel is analyzed. A comprehensive $\mathrm{THz}$ band system model is developed in order to determine the energy consumption of a TRX operating using MQAM and MFSK. Based on the performed analyses, it is deduced that short-range $\mathrm{THz}$ band applications should use MQAM modulation scheme, whereas, medium- and long-range applications should employ MFSK modulation method to minimize the energy consumption per bit. Moreover, optimization of the PHY of a wireless system substantially affects the energy consumption factor. Different PHY parameters are evaluated in order to generate a set of best-functioning parameters which can further reduce the energy consumption factor by $81 \%$. Finally, it is shown that for wireless systems with optimal PHY parameters, the distance at which MFSK performs better than MQAM significantly increases, and consequently for the low- $\mathrm{THz}$ band usage models MQAM is the most optimal modulation scheme.

\section{Acknowledgement}

This work was supported in part by the Scientific and Technological Research Council of Turkey (TUBITAK) under grant \#113E962. An earlier version of this paper was presented in part at the 2017 International Balkan Conference on Communications and Networking (BalkanCom) [51.

\section{References}

[1] World Telecommunication Development Report 2002: Reinventing Telecoms, ITU, Geneva, CHE (2002).

[2] Cisco Visual Networking Index: Global Mobile Data Traffic Forecast Update, 2014-2019, Cisco Systems, San Jose, CA (2015).

[3] Cisco Visual Networking Index: Global Mobile Data Traffic Forecast Update, 2016-2021, Cisco Systems, San Jose, CA (2017).

[4] Resolution ITU-R 56-1: Naming for International Mobile Telecommunications, ITU, Geneva, CHE (2012).

[5] Report ITU-R M.2134: Requirements Related to Technical Performance for IMT-Advanced Radio Interface(s), ITU, Geneva, CHE (2008).

[6] Report ITU-R M.1034-1: Requirements for the Radio Interface(s) for International Mobile Telecommunications-2000 (IMT-2000), ITU, Geneva, CHE (1997).

[7] I. F. Akyildiz, J. M. Jornet, C. Han, Terahertz Band: Next Frontier for Wireless Communications, Physical Communication 12 (0) (2014) 16-32.

[8] I. F. Akyildiz, J. M. Jornet, C. Han, TeraNets: Ultra-broadband Communication Networks in the Terahertz Band, IEEE Wireless Commun. 21 (4) (2014) 130-135.

[9] C. Han, I. F. Akyildiz, Distance-aware Multi-carrier (DAMC) Modulation in Terahertz Band Communication, in: IEEE Int. Conf. on Communications (ICC), 2014, pp. 5461-5467.

[10] S. Cui, A. J. Goldsmith, A. Bahai, Energy-constrained Modulation Optimization, IEEE Trans. on Wireless Commun. 4 (5) (2005) 2349-2360.

[11] T. Yilmaz, O. B. Akan, State-of-the-art and Research Challenges for Consumer Wireless Communications at $60 \mathrm{GHz}$, IEEE Trans. Consum. Electron. 62 (3) (2016) 216-225.

[12] T. Yilmaz, O. B. Akan, Millimeter-wave Communications for 5G Wireless Networks, CRC Press, 2016, pp. 425-440.

[13] T. Yilmaz, O. B. Akan, Attenuation Constant Measurements of Clear Glass Samples at the Low Terahertz Band, IET Electron. Lett. (2018). 
[14] N. Khalid, O. B. Akan, Experimental Throughput Analysis of Low-THz MIMO Communication Channel in 5G Wireless Networks, IEEE Wireless Commun. Lett. 5 (6) (2016) 616-619.

[15] M. Uzunkol, O. D. Gurbuz, F. Golcuk, G. M. Rebeiz, A $0.32 \mathrm{THz}$ SiGe $4 \times 4$ Imaging Array Using High-efficiency On-chip Antennas, IEEE J. Solid-State Circuits, 48 (9) (2013) 2056-2066.

[16] G. Karolyi, D. Gergelyi, P. Foldesy, Sub-THz Sensor Array with Embedded Signal Processing in $90 \mathrm{~nm}$ CMOS Technology, IEEE Sensors J., 14 (8) (2014) 2432-2441.

[17] Q. J. Gu, $\mathrm{THz}$ Interconnect: The Last Centimeter Communication, IEEE Commun. Mag., 53 (4) (2015) 206-215.

[18] W. H. Syed, G. Fiorentino, D. Cavallo, M. Spirito, P. M. Sarro, A. Neto, Design, Fabrication, and Measurements of a $0.3 \mathrm{THz}$ On-chip Double Slot Antenna Enhanced by Artificial Dielectrics, IEEE Trans. Terahertz Science Technol., 5 (2) (2015) 288-298.

[19] T. Yilmaz, O. B. Akan, On the Use of Low Terahertz Band for 5G Indoor Mobile Networks, Computers \& Electrical Engineering 48 (2015) 164-173.

[20] T. Yilmaz, O. B. Akan, On the 5G Wireless Communications at the Low Terahertz Band, arXiv preprint arXiv:1605.02606.

[21] H. Ruonan, E. Afshari, A CMOS High-power Broadband 260-GHz Radiator Array for Spectroscopy, IEEE J. Solid-State Circuits, 48 (12) (2013) 3090-3104.

[22] Y. J. Chen, T. S. Chu, 2-D Direct-coupled Standing-wave Oscillator Arrays, IEEE Trans. Microw. Theory Tech., 61 (12) (2013) 4472-4482.

[23] J. Grzyb, Z. Yan, U. R. Pfeiffer, A 288-GHz Lens-integrated Balanced Triple-push Source in a 65-nm CMOS Technology, IEEE J. Solid-State Circuits, 48 (7) (2013) 1751-1761.

[24] U. L. Rohde, A. K. Poddar, J. Schoepf, R. Rebel, P. Patel, Low Noise Low Cost Ultra Wideband N-push VCO, in: IEEE MTT-S Int. Microwave Symp. Digest, 2005, 1171-1174.

[25] Y. M. Tousi, O. Momeni, E. Afshari, A Novel CMOS High-power Terahertz VCO Based on Coupled Oscillators: Theory and Implementation, IEEE J. Solid-State Circuits, 47 (12) (2012) 3032-3042.

[26] K. Shinwon, C. Jun-Chau, A. M. Niknejad, A W-band Low-noise PLL with a Fundamental VCO in SiGe for Millimeter-wave Applications, IEEE Trans. Microw. Theory Tech., 62 (10) (2014) 2390-2404.

[27] Y. Tousi, E. Afshari, A High-power and Scalable 2-D Phased Array for Terahertz CMOS Integrated Systems, IEEE J. Solid-State Circuits, 50 (2) (2015) 597-609.

[28] K. Sengupta, A. Hajimiri, A 0.28 THz Power-generation and Beam-steering Array in CMOS Based on Distributed Active Radiators, IEEE J. Solid-State Circuits, 47 (12) (2012) 3013-3031.

[29] K. Sengupta, A. Hajimiri, Distributed Active Radiation for $\mathrm{THz}$ Signal Generation, in: IEEE Int. Solid-State Circuits Conf. Digest of Technical Papers (ISSCC), 2011, pp. 288-289.

[30] P. Jung-Dong, K. Shinwon, A. M. Niknejad, A $0.38 \mathrm{THz}$ Fully Integrated Transceiver Utilizing a Quadrature Push-Push Harmonic Circuitry in SiGe BiCMOS, IEEE J. Solid-State Circuits, 47 (10) (2012) 2344-2354.

[31] N. Khalid, N. A. Abbasi, O. B. Akan, $300 \mathrm{GHz}$ Broadband Transceiver Design for Low-THz Band Wireless Communications in Indoor Internet of Things, in: IEEE Int. Conf. on Internet of Things (iThings), 2017, pp. 770-775.

[32] N. Khalid, N. A. Abbasi, O. B. Akan, Capacity and Coverage Analysis for FD-MIMO Based THz Band 5G Indoor Internet of Things, in: IEEE 28th Annu. Int. Symp. on Personal, Indoor, and Mobile Radio Communications (PIMRC), 2017, pp. 1-7.

[33] A. Chakraborty, S. Trotta, J. Wuertele, R. Weigel, A D-band Transceiver Front-end for Broadband Applications in a $0.35 \mu \mathrm{m}$ SiGe Bipolar Technology, in: IEEE Radio Frequency Integrated Circuits Symp., 2014, pp. 405-408.

[34] T. Itoh, G. Haddad, J. Harvey, RF Technologies for Low-Power Wireless Communications, Wiley-IEEE Press, 2001.

[35] S. Cui, A. J. Goldsmith, A. Bahai, Modulation Optimization
Under Energy Constraints, in: IEEE Int. Conf. on Communications (ICC), 2003, vol. 4, pp. 2805-2811.

[36] A. Goldsmith, Wireless Communications, Cambridge University Press, 2005

[37] C. H. Li, et al., A 37.5-mW 8-dBm-EIRP $15.5^{\circ}$-HPBW 338-GHz Terahertz Transmitter Using SoP Heterogeneous System Integration, IEEE Trans. Microw. Theory Tech. 63 (2) (2015) 470-480.

[38] T. Yilmaz, G. Gokkoca, O. B. Akan, Millimetre Wave Communication for 5G IoT Applications, Springer International Publishing, Cham, 2016, pp. 37-53.

[39] B. Thomas, A. Maestrini, G. Beaudin, A Low-noise Fixed-tuned 300-360-GHz Sub-harmonic Mixer Using Planar Schottky Diodes, IEEE Microw. Wireless Compon. Lett. 15 (12) (2005) 865-867.

[40] L. A. Samoska, An Overview of Solid-State Integrated Circuit Amplifiers in the Submillimeter-Wave and $\mathrm{THz}$ Regime, IEEE Trans. Terahertz Science Technol., 1 (1) (2011) 9-24.

41] S. Diebold, et al., A Novel $1 \times 4$ Coupler for Compact and High-Gain Power Amplifier MMICs Around $250 \mathrm{GHz}$, IEEE Trans. Microw. Theory Tech. 63 (3) (2015) 999-1006.

[42] N. Khalid, T. Abbas, M. Ihsan, Power Amplifier Design Using GaN HEMT in Class-AB Mode for LTE Communication Band, in: Int. Wireless Communications and Mobile Computing Conf. (IWCMC), 2015, pp. 685-689.

[43] T. Yilmaz, E. Fadel, O. B. Akan, Employing 60 GHz ISM Band for $5 \mathrm{G}$ Wireless Communications, in: IEEE Int. Black Sea Conf. on Commun. and Networking (BlackSeaCom), 2014, pp. 77-82.

[44] T. Yilmaz, O. B. Akan, Utilizing Terahertz Band for Local and Personal Area Wireless Communication Systems, in: IEEE 19th Int. Workshop on Computer Aided Modeling and Design of Commun. Links and Networks (CAMAD), 2014, pp. 330-334.

45] T. Yilmaz, O. B. Akan, On the Use of the Millimeter Wave and Low Terahertz Bands for Internet of Things, in: IEEE 2nd World Forum on Internet of Things (WF-IoT), 2015, pp. $177-180$

[46] T. Yilmaz, Advanced Image Coding Algorithms: Beyond JPEG2000, MSc thesis, Dept. Electron. Elect. Eng., Univ. College London, London, United Kingdom, (2009).

[47] X. Deng, Y. Li, J. Li, W. Wu, Y. Z. Xiong, A $340 \mathrm{GHz}$ Fully Integrated Transmitter for High-speed Communications, in: Int. Symp. on Integrated Circuits (ISIC), 2014, pp. 300-303.

[48] A. Tang, Y. Kim, M. C. F. Chang, A 65 nm CMOS $330 \mathrm{Mb} / \mathrm{s}$ Microwave Backscatter Link at 2.4 to $2.9 \mathrm{GHz}$ with Ambient Blocker Cancellation, IEEE Microw. Wireless Compon. Lett. 26 (1) (2016) 61-63.

[49] D. J. Broek, E. A. M. Klumperink, B. Nauta, A Self-interference Cancelling Front-end for In-band Full-duplex Wireless and Its Phase Noise Performance, in: IEEE Radio Frequency Integrated Circuits Symp. (RFIC), 2015, pp. 75-78.

[50] B. Debaillie, et al., In-band Full-duplex Transceiver Technology for 5G Mobile Networks, in: 41st European Solid-State Circuits Conf. (ESSCIRC), 2015, pp. 84-87.

[51] N. Khalid, T. Yilmaz, O. B. Akan, Energy-efficient Modulation Scheme for THz-band 5G Femtocell Internet of Things, in: Int. Balkan Conf. on Communications and Networking (BalkanCom), 2017. 